\title{
BMJ Open Role, structure and effects of medical tourism in Africa: a systematic scoping review protocol
}

\author{
John JO Mogaka, Joyce M Tsoka-Gwegweni, Lucia M Mupara, \\ Tivani Mashamba-Thompson
}

To cite: Mogaka JJO, TsokaGwegweni JM, Mupara LM, et al. Role, structure and effects of medical tourism in Africa: a systematic scoping review protocol. BMJ Open 2017;7:e013021. doi:10.1136/ bmjopen-2016-013021

- Prepublication history and additional material for this paper are available online. To view these files please visit the journal online (http://dx.doi. org/10.1136/bmjopen-2016013021).

Received 18 June 2016 Revised 10 0ctober 2016 Accepted 16 January 2017

CrossMark

Discipline of Public Health Medicine, School of Nursing and Public Health, University of KwaZulu Natal, Durban, South Africa

Correspondence to Professor Joyce M TsokaGwegweni; Tsokagwegweni@ ukzn.ac.za

\begin{abstract}
Introduction Some patients travel out of, while others come into Africa for medical care through a growing global phenomenon referred to as medical tourism (MT): the travel in search of medical care that is either unavailable, unaffordable or proscribed at home healthcare systems. While some castigate MT as promoting healthcare inequity, others endorse it as a revenue generator, promising local healthcare system strengthening. Currently, however, the understanding of this component of healthcare in Africa is inadequate. This study seeks to determine the level of knowledge on the role, structure and effect of MT in Africa as it relates to healthcare systems in the region.

Methods Conduct a systematic scoping review to outline the role, structure and effect of MT in Africa. Databases: Academic Search Complete, Business Source Complete. Studies mapped in two stages: (1) mapping the studies based on the relevance of their titles and subject descriptors; (2) applying further inclusion criteria on studies from stage 1. Two reviewers will independently assess study quality and abstract data. Both quantitative and qualitative data analysis will be performed, using STATA V.13 and NVIV0, respectively.

Ethics and dissemination The study results will be disseminated by publication in peer-reviewed journals and findings presented at academic and industry conferences related to MT, public health, health systems strengthening and tourism.

Discussion MT spurs cutting-edge medical technologies, techniques and best practices in healthcare delivery. The two-tier healthcare landscape in Africa, however, presents an exceptionally unique context in which to situate this study. Much has been written about MT globally, but not much is known about the phenomenon in Africa; hence the appropriateness of this scientific assessment of MT in the region. By elucidating the role, structure and effect of this phenomenon, this study hopes to contribute to health systems strengthening in Africa.
\end{abstract}

PROSPERO registration number CRD42016039745.

\section{INTRODUCTION}

Africa is concurrently a source and destination of patients who form part of a global phenomenon referred to as health tourism or medical tourism (MT),${ }^{1-4}$ the practice of travelling abroad to consume healthcare that is either too delayed, unavailable,

\section{Strengths and limitations of this study}

This study offers the 'big picture' analysis of medical tourism (MT) in Africa by synthesising vast amounts of literature on the subject.

- The scoping review approach offers an ideal platform for synthesising literature on MT in Africa whose methodological approaches, settings, study populations and behaviours are wide ranging.

- This approach of synthesising literature, however, presents a limitation in that a detailed analysis of case-specific interventions and quality assessment of individual studies is not applicable.

- As the study will only include literature published in English, studies published in other languages will be omitted. However, research shows that almost $70 \%-90 \%$ of published works are in English. This fact invariably minimises the adverse effects of searching publications written only in English.

- To our knowledge, there is paucity of scientific study on MT in Africa. While this may be a possible limitation in terms of the amount of data for this scoping review, it may be an important finding of this study and a basis for calling for more research in the area.

unaffordable or proscribed at own home countries. ${ }^{15}$

MT challenges the role played by traditional, nation-state-bound healthcare delivery systems. Public health core functions of assessment, policy development and assurance with regard to the health of citizens within the nation-state are fundamentally affected by developments and expansion of MT. $^{6} 7$

On the one hand, MT affords patients individual solution to what is traditionally considered a government concern, health for its citizens, ${ }^{8}$ thereby, arguably, creating more equitable healthcare options for individuals. The wealthy middle class in Africa, for instance, regularly seek advanced medical care abroad. ${ }^{9}$ 


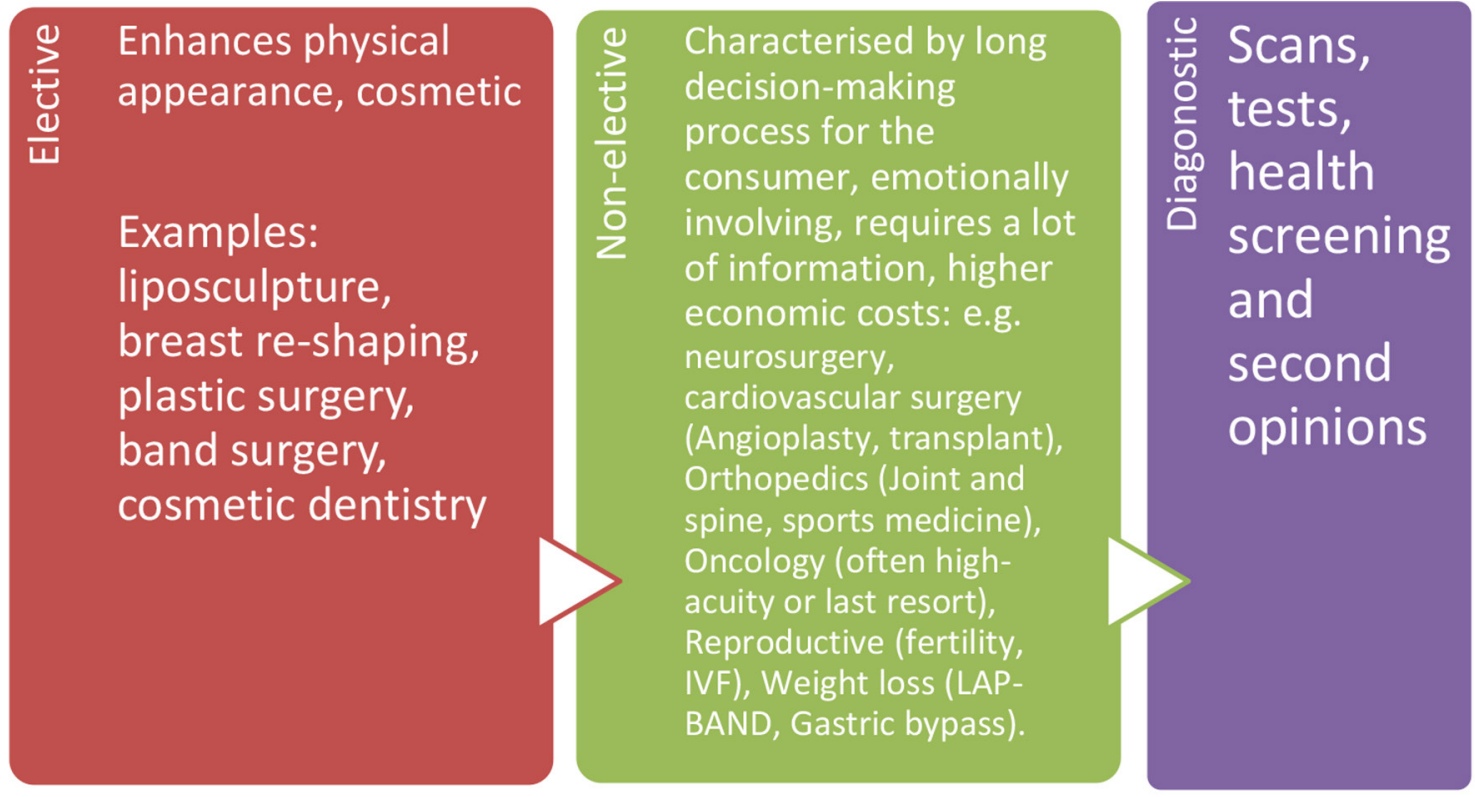

Note: The line between elective and non-elective procedures sometimes is thin. Some procedures might be either side depending on whether the procedure is meant to save or merely enhance life

Figure 1: Medical Procedures and services sought by medical tourists.

Conversely however, MT can indicate a breakdown of service delivery at home health systems. Patients may lack confidence in the ability of home health systems to meet their medical needs and move abroad in relatively large numbers. ${ }^{6} 10$

Whereas MT may pose potentially attractive economic benefits to most governments, especially in Africa, it may particularly prove challenging to local health systems, whose officials may be tasked with both its regulation domestically and promotion internationally, while at the same time grappling with risks such endeavours pose to the national health system. ${ }^{8}$

Specialised medical services and procedures offered to medical tourists include elective, non-elective and diagnostic as depicted in figure 1 .

However, current availability, pricing and geodistribution of these procedures and services in Africa is not sufficiently known.

Principally, MT entails highly trained and experienced physicians, high-tech medical equipments and specialised ultra-modern medical facilities. Some African countries are said to have invested in or attracted ample pool of specialist physicians in quality private hospitals whose medical facilities are similar to the ones in developed countries. ${ }^{9}$ Costs associated with these MT infrastructure are substantial. ${ }^{11}$ Similarly, MT is said to cause competition for best local resources including qualified medical professionals $^{11}{ }^{12}$ and transplant organs. The accrued benefits to the local host population, in turn, is however, not well-known.
Many arguments have been advanced for and against MT in most LMICs, including Africa. ${ }^{11}$ figure 2 summarises these advantages and disadvantages.

The extent and dimension of these pros and cons in as far as healthcare systems in Africa are concerned, however, is inexact. ${ }^{2581113-16}$

The role and effects of MT in Africa need to be investigated and documented in the face of Africa's two-tier healthcare systems, with a relatively efficient private, juxtaposed with a relatively overburdened public medical care sector.

MT services are primarily provided by the private sector and payments are mostly out of pocket. Whereas private healthcare providers have little incentive to consider population-wide and -based services, the public at large must be served through public health interventions focused on the health needs of the entire population or population groups. Individual healthcare, population health and public policy decisions must, therefore, be premised on best available evidence to avoid poor and inappropriate interventions. ${ }^{3}{ }^{14}{ }^{16-19}$ However, for MT in Africa, this is clearly difficult because of paucity of scientific evidence on the subject. Moreover, even with the available evidence, the information generated from individual studies may be biased, methodologically flawed, time and context constrained, resulting in conflicting conclusions. This does not allow MT in Africa to be understood well. ${ }^{20}$ Hence, the necessity and justification for this scoping review, whose purpose is to assess current level of knowledge on MT in Africa with specific reference to Africa's healthcare systems; 


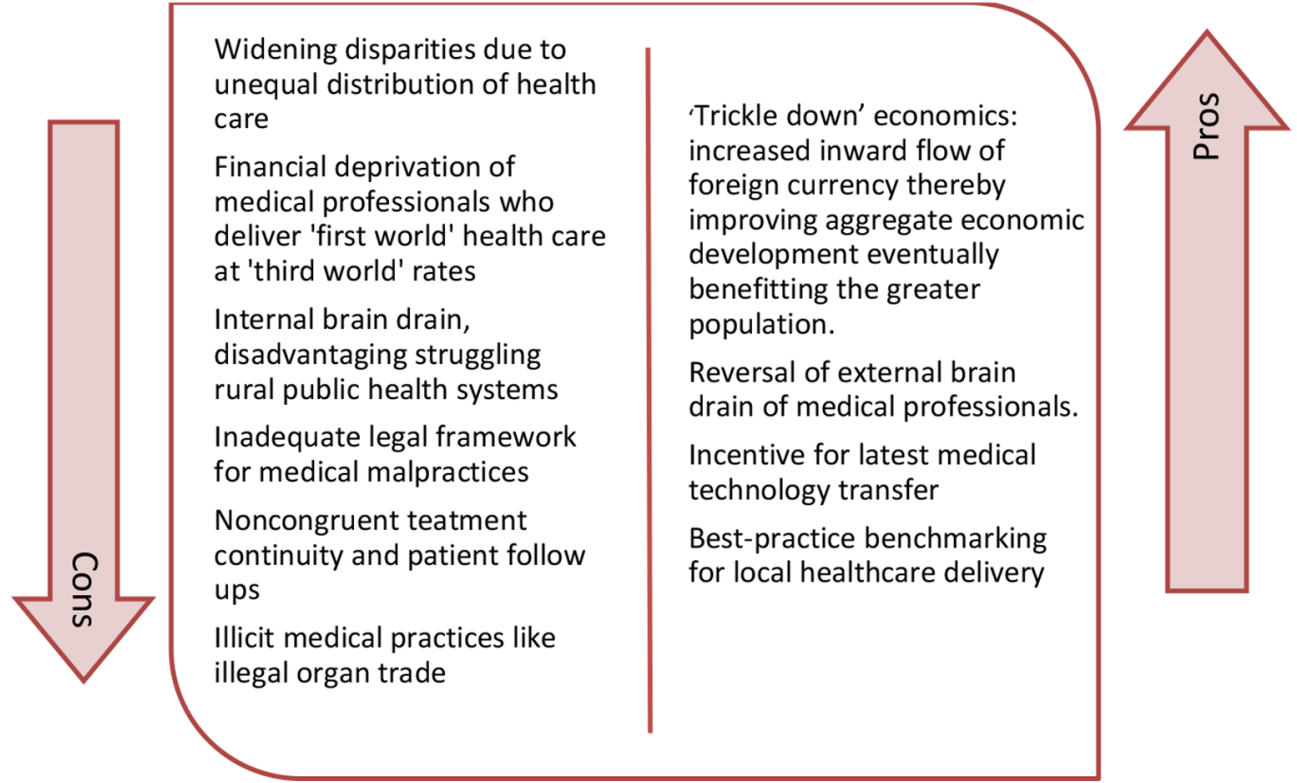

Figure 2 Advantages and disadvantages of medical tourism in Africa. (Source: adapted from Bookman and Bookman). ${ }^{11}$

by systematically reviewing available literature on the subject. The study will focus on MT flows out of, into and within Africa.

In this study, we take Africa geoscheme approach used by the African Union and United Nations in dividing the region directionally ${ }^{21}$ :

- North Africa lies north of the Sahara and runs along the Mediterranean coast.

- West Africa excludes Northern Africa and the Maghreb and includes the large portions of the Sahara Desert and the Adamawa Mountains.

- East Africa stretches from the Horn of Africa to Mozambique, including Madagascar, Seychelles and Mauritius.

- Central Africa is the large mass at the centre of Africa which either does not fall squarely into any other region or only partially does so.

- Southern Africa consists of the portion generally south of $-10^{\circ}$ latitude and the great rainforests of Congo.

The main aim of this study is to outline the role, structure and effect of MT in Africa as it relates to Africa's healthcare systems by answering the following specific questions:

i. What is published about Africa's MT infrastructure: the availability, pricing and geodistribution of specialised medical procedures and services offered to medical tourists in Africa?

ii. Who are the main MT actors in Africa, as identified in the literature?

iii. What are the identified effects of MT on healthcare systems in Africa?

iv. What are the identified ethical issues associated with MT in Africa?

\section{METHODS}

The study will employ Arksey and O'Malley scoping review framework as well as incorporate recent scoping review suggestions by Levac et al. ${ }^{22} 23$

\section{Identification of relevant studies}

Key terms

Multiple terms are used to refer to MT in literature. Evidence indicate that while literature on MT is generally growing, it is mostly focused either on individual case studies or specific aspects of MT. ${ }^{10}$ Therefore, an extensive list of primary and secondary search terms will be developed. Search terms will include: MT, stem cell tourism, fertility tourism, dental tourism, health tourism and transplant tourism, among other terms with Africa as the bounding parameter. The university librarian will help in finalising the keyword and search strategy in this study. Attempt will be made to obtain relevant documents that are not readily available through concerned authors or publishers.

\section{Databases}

Academic databases: Academic Search Complete, Business Source Complete, PsycARTICLES (EBSCO), PsycINFO (EBSCO), Health Source-Consumer Edition, Health Source-Nursing/Academic Edition and Sabinet.

Search engines: Google and Google Scholar.

Relevant MT industry associations, Organisation for Economic Co-operation and Development, WHO, World Bank and other multilateral organisations' websites will be used to search for government reports, practice guidelines and industry reports.

Relevant research dissertations will be searched through WorldCat via Online Computer Library center(OCLC) and reference list scanning of included studies. 
Table 1 Electronic search record (source: adapted from Mashamba-Thompson et al) ${ }^{24}$

\begin{tabular}{llll}
\hline Keyword & $\begin{array}{l}\text { Search engine } \\
\text { used }\end{array}$ & $\begin{array}{l}\text { Number of } \\
\text { publications } \\
\text { retrieved }\end{array}$ \\
\hline
\end{tabular}

\section{Search strategy}

The databases selected will cover a broad range of disciplines to ensure sensitivity. Search queries will be tailored to specific requirements for each database. For academic databases, keywords will be combined using Boolean operators (AND, OR, NOT). A snowball technique will be used to find related works.

However, since this will be an iterative process, the detailed search strategy will be documented in the analysis and write up of the full review.

Researchers will keep an updated record on dates and the numbers of publications identified during each session of literature search using a search records table ${ }^{24}$ as shown in table 1 .

\section{Study selection (screening)}

A two-stage screening process will be used to assess the relevance of studies identified in the search. Two reviewers will independently evaluate and apply the identified selection criteria to candidate literature titles and abstracts. Titles and abstracts will be screened as 'include', 'exclude' or 'uncertain'. Full text of articles screened as 'uncertain' will be reviewed by the third reviewer for verification against the inclusion criteria. During the first stage, only the title and abstract of citations will be reviewed to preclude articles that do not meet the minimum inclusion criteria. A title and abstract relevance screening form will be developed and pretested on a convenience sample of 10 academic citations to evaluate reviewer agreement. A kappa calculation will be done based on the results of this pretest. This will in turn be used to show the reviewers' inter-rater agreement level. Generally, a kappa score over 0.8 is considered a high level of agreement. ${ }^{25}$ In accordance with recommendations by Levac et al, ${ }^{23}$ after reviewing every batch of 20 to 30 publications, the reviewers will meet to resolve any conflicts and ensure consistency with the research question and purpose.

Executive summaries in grey literature will be treated as abstracts. Relevant titles whose abstracts are not available will be taken to screening stage 2 for full review. During screening stage 2, reviewers will independently screen the rest of the search results using the predefined inclusion-exclusion criteria. Any ensuing discrepancies will be resolved by discussion or the involvement of the third reviewer.
To capture and present the screening process, the Preferred Reporting Items for Systematic and Meta-Analyses flow diagram in figure 3 will be used.

\section{Inclusion criteria}

- Evidence published in English.

- There will be no publication date restrictions up to 06 June 2016.

- Literature with substantial focus on MT in Africa including: peer-reviewed journal articles, systematic reviews, scoping reviews, meta-analysis and rapid reviews, government and non-governmental organisation reports and academic dissertations.

- Research focusing on MT in low-income and middle-income countries and whose conclusions and discussion demonstrate transferable findings to African settings.

- All study designs will be considered including qualitative, quantitative and mixed-methods studies.

- Studies focusing on healthcare provision through specified bilateral or multilateral government agreements in Africa.

\section{Exclusion criteria}

- Evidence focusing on MT outside Africa and whose results are non-transferable to African settings.

- Evidence focusing on people forced to seek emergency medical care in conflict or postconflict settings as opposed to organised medical travel in non-conflict settings.

- Evidence with focus on emergency medical care for conventional tourists.

- Evidence where medical care provision to medical tourists is not explicitly differentiated from the dayto-day provision of healthcare offered to the general public.

- Evidence with main focus on wellness tourism.

\section{Charting the evidence (data abstraction)}

After title and abstract screening, successful candidate citations will be exported to endnote bibliographic dataset for subsequent full-text review. Endnote library application will be used to discard any duplicates. A data abstraction spreadsheet will be developed collectively by the reviewers to extract predetermined variables and themes. Structuring this spreadsheet database will involve selecting and defining data categories and subcategories, as advised by the MT conceptual framework..$^{20}$ It will be secured online so that involved reviewers will have access and can make updates freely.

Bibliographic details, study design, number of participants, intervention(s), comparison(s), study setting, funding source and conclusions for the primary and secondary outcomes of interest will be extracted. This dataset will be populated from each selected paper. This step will be done iteratively as more familiarity of literature is gained and revisions done as appropriate. See 


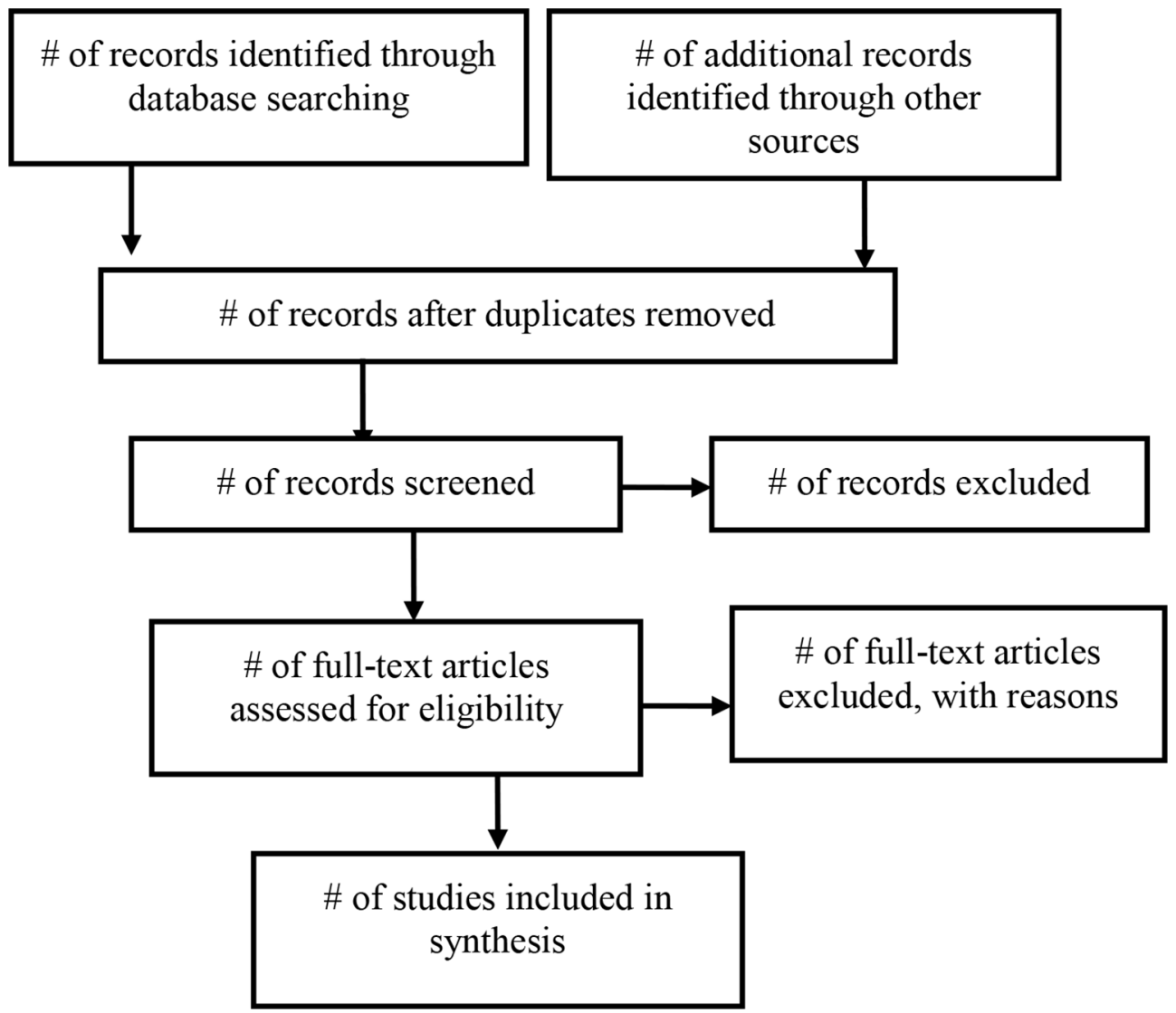

Figure 3 Preferred Reporting Items for Systematic and Meta-Analyses flow diagram. \# stands for number. (Source: adapted from Moher et al). ${ }^{47}$

online supplementary appendix I for the data extraction form.

\section{Risk of bias (quality) assessment of individual studies}

Methodological quality of quantitative, qualitative and mixed-method primary studies will be assessed using the Mixed Methods Appraisal Tool. ${ }^{26}$ Studies will not be excluded on account of low-quality scores, but quality scores will be reported and considered in the narrative synthesis of the evidence.

\section{Collating, summarising and reporting the data}

The extracted data will be summarised and presented. This is in line with the purpose of a scoping review which is geared towards establishing the scope of the current evidence, summarising the results as presented across articles and not synthesising or distilling specific results. ${ }^{22} 2327$ The data will be analysed accordingly to address the main aim and the four specific study questions. Further to this, the study team will scrutinise the meanings of the findings as they relate to overall purpose of the study, discuss the implications for future research, practice and policy.

Data analysis will employ both quantitative and qualitative methods.

After the coding and validation of the spreadsheet file, the data will be exported into STATA V.13 for analysis. Descriptive statistics will be used to summarise the data. Frequencies and percentages will be used to describe nominal data. A basic statistical account of the amount, type, and distribution of the studies included in the review will be presented.

Also, a thematic analysis and concept map of the results will be presented. Thematic analysis will be carried out using NVIVO research software.

This analysis will generally answer questions like: how large is the literature on MT in Africa (number of papers), when was it published (age), where was the research conducted (geographic distribution), at what scale and by whom (geographic/institution); where, when and by whom research was published; the geographical spread of the research; types of methods used; types of subjects examined; types of variables measured; different disciplines assessing the topic and patterns found in research results. We will use statistical means to produce tables and charts that depict cross-tabulations like: MT in Africa as it relates to study designs used, type of treatments, medical facilities and selected patient characteristics (such as age, sex, geography, ethnicity).

\section{DISCUSSION}

MT is different from medical care administered to conventional tourists in need of emergency medical care. Unlike the latter, the former specifically refers to 'foreign patients' who travel abroad for the express purpose of obtaining medical care. ${ }^{16}$ MT is defined as a 
set of socioeconomic activities carried out either by or for medical tourists. ${ }^{28}$ The activities carried out by medical tourists correspond to the travel of patients in search of health services outside the jurisdiction of their home health systems, ${ }^{20}$ while those carried out for them correspond to attempts on the part of destinations to attract international patients by promoting their healthcare services and facilities. ${ }^{20}$ Although there is no standard meaning that is assigned to it, the term 'tourism' could refer to the measures taken by destination countries (supply side) to attract and meet the needs of international patients. The term could also refer to the tourism channels the medical tourist uses to get to the destination countries.

In light of the above, therefore, evidence with focus on emergency medical care for conventional tourists will be excluded.

This study will include literature on formal bilateral and multilateral medical care agreements that enable patients to be attended to in other countries, but exclude those papers that focus on 'forced' cross-border medical care, especially if public health facilities of destination countries are 'forced' to provide the care on humanitarian grounds. Such conditions exist especially in conflict and postconflict zones.

Evidence on MT that is global in outlook will be included in-as-far-as its findings are relevant to Africa and its healthcare systems. Relevance will be informed as progressive familiarity with literature is gained in the study.

Africa presents an exceptionally unique and fascinating context in which to situate this MT study. This is because of the stark contrast in the African healthcare landscape: whereas MT demands highly specialised medics, capable of executing most complicated surgeries, clad in advanced medical technologies and luxury, specialised hospitals; Africa, on the other hand, has a large local population enduring rudimentary health, insufficient clean water and inadequate sanitation.

To our knowledge, this is the first systematic scoping review that attempts to expound on the role, structure and effects of MT as it relates to healthcare systems in Africa. Most MT evidence on Africa is emergent and multidisciplinary in nature, hence the critical necessity of a scoping review to map the range of extant evidence and systematically identify research gaps to more clearly illumine the role, structure and effects of MT in Africa.

It is anticipated that while findings from this study will lead to clearer understanding of MT in Africa, they will also contribute to the resolving of paradoxical healthcare issues in the region. The needs of 'foreign' patients may be prioritised over those of locals, especially if economic incentives over weigh public health considerations, thereby unfairly shifting resources towards the development of more expensive clinical interventions that cater for exclusive few, while promoting healthcare inequity. ${ }^{6} 829$ For instance, reproductive tourism uses expensive and non-routine medical expertise to bring new children into this world, ${ }^{30} 31$ yet yearly, malaria, pneumonia and other preventable infections kill millions of under-fives in Africa. ${ }^{32} 33$

MT is promoted as a solution to healthcare queues in some parts of the world. ${ }^{1911131534}$ Able-to-pay patients opt to fly to Africa to avoid these long queues to get faster service. But, in sharp contrast, local patients are forced to experience similar long queues in oversubscribed and underfunded local health systems. ${ }^{9} 33$ 35-38

Some literature suggest that MT has been growing globally. ${ }^{11} 3940$ This growth, however, seems to be based solely on MT advantages to medical tourists, short-term economic rent for destination economies and profits to care providers. Medical tourists benefit from preferential treatment based on their ability to pay for medical services. ${ }^{20}$ Destination countries benefit in revenue generation. ${ }^{114142}$

Motivated by these benefits, many African countries are competing for the global MT dollar. ${ }^{43-45}$ Unfortunately, MT in most of these countries is based on unsustainable, haphazard regulatory frameworks. ${ }^{46}$

Given its potential contextual significance, it is imperative that a scientific reconnaissance study be carried out on MT in Africa. This scoping study is, therefore, an attempt to do this by providing more information about MT in Africa to policy makers, healthcare providers, potential patients and future researchers. This study will therefore o contribute to improved healthcare systems in Africa.

Correction notice This paper has been amended since it was published Online First. Owing to a scripting error, some of the publisher names in the references were replaced with 'BMJ Publishing Group'. This only affected the full text version, not the PDF. We have since corrected these errors and the correct publishers have been inserted into the references.

Acknowledgements We would like to thank the College of Health Sciences, University of KwaZulu-Natal for providing support in carrying out this study. Furthermore, we would like to thank Duke Magwaza, UKZN librarian, for his support in database search strategy.

Contributors JJOM and JMTG conceptualised the study and developed the background. LMM and JJOM prepared the draft of the research proposal. JJOM and TMT designed the study and contributed to developing methods relating to review and synthesis of data. All authors planned the output of the review, reviewed draft versions of the manuscript and approved the final version of the manuscript.

Competing interests None declared.

Provenance and peer review Not commissioned; externally peer reviewed.

Open Access This is an Open Access article distributed in accordance with the Creative Commons Attribution Non Commercial (CC BY-NC 4.0) license, which permits others to distribute, remix, adapt, build upon this work non-commercially, and license their derivative works on different terms, provided the original work is properly cited and the use is non-commercial. See: http://creativecommons.org/ licenses/by-nc/4.0/

(c) Article author(s) (or their employer(s) unless otherwise stated in the text of the article) 2017. All rights reserved. No commercial use is permitted unless otherwise expressly granted.

\section{REFERENCES}

1. Alsharif MJ, Labonté R, Zuxun Lu. Patients beyond borders: A study of medical tourists in four countries. Glob Soc Policy 2010;10:315-35. 
2. Hall CM. Health and medical tourism: a kill or cure for global public health? Tourism Review 2011;66:4-15.

3. Johnston R, Crooks VA, Snyder J, et al. What is known about the effects of medical tourism in destination and departure countries? A scoping review. Int J Equity Health 2010;9:24.

4. Turner LG. Quality in health care and globalization of health services: accreditation and regulatory oversight of medical tourism companies. Int J Qual Health Care 2011;23:1-7.

5. Reddy SG. Medical tourism in India: an exploratory study. 2013 Kansas State University Kansas.

6. Dominique M. Medical Tourism and Cross-border Care 2013.

7. Handler A, Issel M, Turnock B. A conceptual framework to measure performance of the public health system. Am J Public Health 2001;91:1235-9.

8. Pocock NS, Phua KH. Medical tourism and policy implications for health systems: a conceptual framework from a comparative study of Thailand, Singapore and Malaysia. Global Health 2011;7:121.

9. Ahwireng-Obeng $F$, van Loggerenberg $\mathrm{C}$. Africa's middle class women bring entrepreneurial opportunities in breast care medical tourism to South Africa. Int J Health Plann Manage 2011;26:39-55.

10. Hanefeld J, Lunt N, Smith R, et al. Why do medical tourists travel to where they do? the role of networks in determining medical travel. Soc Sci Med 2015;124:356-63.

11. Bookman MZ, Bookman KR. Medical Tourism in Developing Countries 2007.

12. Turner L. 'First World Health Care at Third World Prices': Globalization, Bioethics and Medical Tourism. Biosocieties 2007;2:303-25.

13. Connell J. Contemporary medical tourism: conceptualisation, culture and commodification. Tour Manag 2013;34:1-13.

14. Crush J, Chikanda A. South-South medical tourism and the quest for health in Southern Africa. Soc Sci Med 2015;124:313-20.

15. Crush J, Chikand A, Maswikwa B, et al. South-South and NorthSouth medical tourism: the case of South Africa.. Travelling well: Essays in medical tourism. Ottawa Ottawa : University of Ottawa, Institute of Population Health, 2013:43-61.

16. Lunt N, Hardey M, Mannion R. Nip, tuck and click: medical tourism and the emergence of web-based health information. Open Med Inform J 2010;4:1-11.

17. Abdullahel $\mathrm{H}, 2009$. Globalization, medical tourism and health equity. Symposium on Implications of Medical Tourism forCanadian Health and Health Policy, Ottawa.

18. Crooks VA, Kingsbury P, Snyder J, et al. What is known about the patient's experience of medical tourism? A scoping review. BMC Health Serv Res 2010;10:10 266.

19. Runnels V, et al. 12 conclusions: medical Tourism today and tomorrow, in Transdisciplinary Studies in Population Health Series. Chapt.

20. Heung VCS, Kucukusta D, Song H. A Conceptual Model of Medical Tourism: implications for Future Research. Journal of Travel \& Tourism Marketing 2010;27:236-51.

21. Division UNS. Standard Country and Area Codes classifications (M49)2016 http://unstats.un.org/unsd/methods/m49/m49regin.htm

22. Arksey H, O'Malley L. Scoping studies: towards a methodological framework. Int J Soc Res Methodol 2005;8:19-32.

23. Levac D, Colquhoun H, O'Brien KK. Scoping studies: advancing the methodology. Implement Sci 2010;5:1-9.

24. Mashamba-Thompson TP, Sartorius B, Thabane L, et al. Impact of point-of-care diagnostics on maternal outcomes in HIV-infected women: systematic review and meta-analysis protocol. BMJ Open 2016;6:e008002
25. McHugh ML. Interrater reliability: the kappa statistic. Biochem Med 2012;22:276-82.

26. Pluye $\mathrm{P}$, et al. Proposal: a mixed methods appraisal tool for systematic mixed studies reviews. $2011 \mathrm{http} / / / w w w . w e b c i t a t i o n$. org/5tTRTc9yJ: http://mixedmethodsappraisaltoolpublic.pbworks. com

27. Pham MT, Rajić A, Greig JD, et al. A scoping review of scoping reviews: advancing the approach and enhancing the consistency. Res Synth Methods 2014;5:371-85.

28. Han X, Fang B. Measuring the size of tourism and its impact in an economy. Statistical Journal of the UN Economic Commission for Europe 1997;14:357.

29. Gbenga A. 2014. Nigeria: awry tales of Nigerians seeking medical treatment abroad. The Guardian. Nigeria.

30. ProductionsDL. In South Africa, fertility treatments are available at a number of world-class clinics that specialise in the field. Because of the high costs of fertility treatments internationally, it makes financial sense for couples to come to South Africa and consult with our world-class specialists. $2016 \mathrm{http}: / /$ www.southafrica.net/za/en/ articles/entry/article-southafrica.net-fertility-treatments

31. Team SFLW. 2015High Court considers South African surrogate.Family Law Newswire. http://www.marilynstowe.co.uk/ 2015/06/22/high-court-considers-south-african-surrogate

32. Cooke JG. Public Health in Africa - A Report of the CSIS Global Health Policy Center. J Public Health Africa 2010;1.

33. Kaseje D. Health care in Africa: challenges. opportunities and an emerging model for improvement 2006.

34. Caballero-Danell S, Mugomba C. Medical tourism and its entrepreneurial opportunities: a conceptual framework for entry into the industry. 2007.

35. Africa K. The state of healthcare in africaKPMG: south. Africa 2012.

36. Ajayi IO, Adewole IF. Breast and cervical Cancer screening activities among family physicians in Nigeria. Afr J Med Med Sci 2002;31:305-9.

37. Akukwe C. Healthcare delivery in Africa: issues, choices, challenges and opportunities. . African Renaissance, 2006;3(4).

38. Clemens MA, Pettersson G. New data on african health professionals abroad. Hum Resour Health 2008;6:1-11.

39. George R. Medical tourism: surgeon \& safari. South African travel and tourism cases 2004: 241-243.

40. Herrick DM. Medical tourism: global competition in health care. National Center for Policy Analysis 2007;1.

41. Christie I, et al. Tourism in Africa: harnessing tourism for growth and improved livelihoods: World Bank Publications, 2014.

42. Kachipande S. Medical Tourism in Africa. Sun, Sea, Scalpel and Safari 2013

43. Maaka TP. The role of medical tourism in 21 st century Africa health system. African Rennaissance 2006;3:99-110.

44. Journal IMT. Kenya targeting medical tourism. 2015 http://www.imtj. com/news/kenya-targeting-medical-tourism

45. Uppiah M, et al, 2014. The legal implications for Mauritius to develop the medical tourism sector. 4th Advances in Hospitality \& Tourism Marketing \& Management Con.

46. Mahomed S, Slabbert MN. Stem cell tourism in South Africa: the legal position. South African Journal of Bioethics and Law 2012;5:69-73.

47. Moher D, Liberati A, Tetzlaff J, et al. Reprint--preferred reporting items for systematic reviews and meta-analyses: the PRISMA statement. Phys Ther 2009;89:873-80. 